\title{
On the measurement of energy fluxes in plasmas using a calorimetric probe and a thermopile sensor
}

\author{
Pierre-Antoine Cormier ${ }^{1}$, Marc Stahl' ${ }^{2}$, Anne-Lise Thomann ${ }^{1}$, Rémi Dussart ${ }^{1}$, Matthias Wolter ${ }^{2}$, \\ Nadjib Semmar ${ }^{1}$, Jacky Mathias ${ }^{1}$, Holger Kersten ${ }^{2}$ \\ ${ }^{1}$ Laboratoire GREMI, CNRS/Université d'Orléans, 14 rue d'Issoudun BP 6744, 45067, Orléans cedex2, France \\ ${ }^{2}$ University of Kiel, Institute of Experimental and Applied Physics, Leibnizstr. 11-19, 24098 Kiel, Germany
}

\begin{abstract}
Two different diagnostics for the determination of the energy influx in plasma processes were used to characterize an ion beam source and an asymmetric RF discharge. The related energy fluxes were measured in dependence on the ion energy and on the RF power, respectively. The first sensor, called HFM (Heat Flux Microsensor) is a thermopile which allows for direct energy flux measurements. With the second sensor, a calorimetric probe, the energy influx has been calculated from the temporal temperature evolution preliminary registered. Although the working principle of both sensors is different, the obtained results are in good agreement. In the ion beam $(<1,5 \mathrm{keV})$ ) rather high energy influxes are achieved (up to $700 \mathrm{~mW} / \mathrm{cm}^{2}$ ), whereas the values measured in the asymmetric RF discharge were lower than $50 \mathrm{~mW} / \mathrm{cm}^{2}$ for discharge powers in the range of 10 to $100 \mathrm{~W}$. The performances and limitations of both sensors are compared and discussed.
\end{abstract}

\section{Introduction}

The knowledge of the energy influx (e.g. deposited power) at surfaces (walls, target, substrates etc.) is a key parameter for the control of low pressure plasma processes, especially those dedicated to material processing such as etching, surface treatment or thin film deposition $[1,2]$. To evaluate the energy transferred from a plasma to a surface (surface of the probe in this study), several species (electrons, neutrals, ions) and elementary processes (atom condensation, chemical reactions, radiation etc.) have to be taken in account. The total energy flux $\mathrm{P}_{\mathrm{E}}$ is the surface integral over the sum of the energy flux density $\left(\mathrm{J}_{\text {in }}\right)$ contributions:

$P_{E}=\int J_{i n} \cdot d S=\int\left(J_{i}+J_{e}+J_{n}+J_{\text {rad }}+J_{\text {film }}\right) \cdot d S$

$\mathrm{J}_{\mathrm{i}}, \mathrm{J}_{\mathrm{e}}$ and $\mathrm{J}_{\mathrm{n}}$ are the contributions of ions, electrons and neutrals, respectively. $\mathrm{J}_{\mathrm{rad}}$ regroups the energy influxes induced by radiation from hot surfaces or by plasma emission, and $\mathrm{J}_{\text {film }}$ is the power released during film growth due to condensation, adsorption, and chemical reactions.

The total energy influx can be obtained by different methods. First of all, it can be calculated from equation (1) by carrying out simulation of particle transport through the plasma and of the interaction with the substrate surface [3,4]. Even if this method is based on experimental data (characterization of the plasma), many assumptions have to be done. For instance, the energy effectively released by the particles when they interact with the surface (condensation, bombardment, chemical reaction etc.) is not well known. These calculations only give approximated values of the transferred energy.

Thus, it is of particular interest to perform direct measurements of the deposited energy. This can be done by recording the temperature evolution at the surface [5,6]. Since the 60ies many authors tested various techniques to measure the energy influx [7, 8, 9]. Among them, 
calorimetric probes, based on an original idea of Thornton [7], were successfully applied to plasma science and were developed [10-13]. The energy influx is also calculated from the measurements of the temporal temperature evolution $\mathrm{T}_{\mathrm{S}}(\mathrm{t})$. The calorimetric probe $[2,12]$ designed in the IEAP Kiel consists of a thermocouple brazed to a metal plate (substrate dummy). This probe has been used by Kersten et al to characterize many kinds of low pressure plasmas used for powder generation, space propulsion, PECVD etc. [12, 14]

Another way to determine the energy transfer between plasma and surface is the use of a sensor which is sensitive to the energy flux itself. The Heat Flux Microsensor (HFM) which was firstly explored for plasma diagnostics at GREMI exactly uses this principle $[15,16]$. The microsensor is composed of a Pt100 temperature sensor and a thermopile. According to the Seebeck effect, the thermopile voltage is directly proportional to the energy influx. The researches at the GREMI mainly deal with the understanding of fundamental mechanisms involved in plasma processing of materials. Previous works have shown the great interest of performing energy flux measurements with the HFM to study such mechanisms. For example, it has been possible to detect the energy transferred to the surface by a chemical reaction (silicon etching) or by the condensation of atoms (plasma sputtering deposition) $[15,17]$.

The aim of the present work is to compare performances and limitations of the calorimetric and HFM sensors for measurements in low pressure plasmas. For that, they were alternatively placed in well characterized plasma environments, e.g. in ion beam source [12, 18] and in asymmetric RF discharge [14]. As we will see in the following, both sensors have drawbacks and advantages. We hope that this study will help researchers to choose between them, depending on the kind of measurements they need to perform.

\section{Experimental apparatus}

\subsection{Description of the HFM}

The HFM used is a commercial one, developed by Vattel (Vattel-HFM-7). The sensor with an active area of $17.1 \mathrm{~mm}^{2}$ is shown in Fig 1 . This is a thermopile consisting of 1600 thermocouple junctions per $\mathrm{cm}^{2}$, which provides a very sensitive measurement of the energy influx, with a time resolution in the order of $1 \mathrm{~ms}$. The sensor is based on the Seebeck effect: when two metal-metal junctions are set at different temperatures, a voltage difference appears between them. In the Vattel system the junctions are thin film designed. The small temperature gradient that establishes at the extreme surface, when submitted to an energy influx, induced the creation of a voltage at each metal junction. The voltage delivered by the thermopile is the sum of all junction voltages and is directly proportional to the temperature gradient and thus to the energy flux density. The Pt100 temperature sensor is used to control the temperature of the HFM during the experiment.

This commercial system is inserted in a copper chamber for water cooling. It is kept at $5^{\circ} \mathrm{C}$ during measurements in order to avoid the increase of the sensor temperature, which would lead to radiation loss by IR emission. Hence, due to its sensitivity, it is possible to detect relatively small energy flux contributions. In Fig 2 a typical slope of the voltage obtained when the HFM is exposed to an energy fluxes is presented. The offset of about $2,5 \mu \mathrm{V}$ which corresponds to $6 \mathrm{~mW} / \mathrm{cm}^{2}$ is caused by the radiative flux between the reactor at ambient temperature and the cooled HFM. The voltage is measured with a nanovoltmeter (Keithley 2182) every $0.5 \mathrm{~s}$. Although the duration of a typical measurement in an RF plasma was nearly 2 minutes (Fig. 2), one can clearly recognize that a stable value of the energy flux density is already reached after a short time (less than $5 \mathrm{~s}$ ). In the present configuration the time resolution is limited by the acquisition time, e.g. two measurements per second. 
The HFM has to be calibrated for its use in low pressure plasma environment [16]. This calibration procedure was done in vacuum by known IR radiation emitted from a home- made blackbody and according to a NIST calibration protocol [19]. The black body was placed in front of the active surface of the HFM. For reliable calibration the HFM active area was coated with a thin black paint (zynolithe) film whose emissivity is close to unity, and the Pt100 was not activated in order to avoid self-heating due to Joule effect.

For the experiments presented in this paper a copper plate having a diameter of $6.89 \mathrm{~mm}$ and a thickness of $100 \mu \mathrm{m}$ was stuck on the active area to protect the thermopile against high energy flux irradiation. A thermal paste was used to ensure a good thermal contact between copper plate and thermopile. To check that the bonding of a sample on the HFM does not affect the measurement, calibration was made with and without the copper sample. By using the copper sample the difference between sample and sensor active areas has to be taken in account for the determination of the energy flux density. It can be seen in Fig. 3 that the values of the energy influx in both cases are nearly the same.

\subsection{Design of the calorimetric (thermal) probe}

The calorimetric probe consists of a type-K thermocouple soldered to a tungsten plate with a diameter of $20 \mathrm{~mm}$ and a thickness of $50 \mu \mathrm{m}$, see Fig.4. Details of the probe construction may be found elsewhere [18]. Although an additional copper wire connected to the plate allows for biasing, the experiments were performed at floating potential. The energy flux is calculated from the measured temperature characteristics $T_{S}(t)$ (Fig. 5), e.g. the temperature evolution during the heating process, when the probe is exposed to an energy flux, and the cooling, when the energy source is switched off. For a reliable calculation of $\mathrm{J}_{\text {in }}$ a temperature curve for a certain time of about some minutes has to be acquired. The calculation is based on the rate of change in the temperature of the test substrate $\mathrm{dT}_{\mathrm{S}} / \mathrm{dt}$ given by:

$\dot{H}_{S}=C_{S} \frac{d T_{S}}{d t}$,

where $\mathrm{C}_{\mathrm{S}}$ is the effective heat capacity of the calorimetric probe, and Hs the enthalpy of the substrate.

The energy balance during the heating and the cooling cycles are given by $\dot{H}_{S \text {, heat }}=P_{\text {in }}-P_{\text {out }}\left(T_{S}\right)$ and $\dot{H}_{S, \text { cool }}=-P_{\text {out }}\left(T_{S}\right)$, respectively. Hence, the total incoming power $\left(\mathrm{P}_{\text {in }}\right)$ at the test substrate surface for each temperature is given by:

$P_{i n}=C_{S}\left[\frac{d T_{S, h e a t}}{d t}-\frac{d T_{S, \text { cool }}}{d t}\right]$.

The effective heat capacity of the probe is a key parameter for the correct determination of the absolute energy influx at the probe surface. Since each component of the probe (thermocouple, metal plate, copper wire) has its own heat capacity the calorimetric probe has to be calibrated. The calibration of the probe is carried out with an electron beam according to a protocol developed in the IEAP Kiel [18]. For the present measurements a tungsten probe with an effective heat capacity of $281 \mathrm{~mJ} / \mathrm{K}$ was used.

Because the probe is connected to an electrical circuit without protection, a noisy temperature signal may be provided. This effect was especially the case in RF discharge operation. Therefore, the calorimetric probe was equipped with an additional RF filter.

\subsection{Plasma environments for testing the probes}


At first, the calorimetric probe and the HFM were alternatively placed in the center of a broad beam ion source [12, 20]. An ECR plasma is used as ion source at an operating pressure of $5.10^{-2} \mathrm{~Pa}$. Ions are extracted and accelerated from the plasma by two planar molybdenum grids having a diameter of $125 \mathrm{~mm}$ and composed of 751 circular $3.2 \mathrm{~mm}$ holes. Changing the voltage $\left(\mathrm{U}_{\mathrm{A}}\right)$ of the anode ring permits the control of the ion energy. The distance between the grids and the sensor (calorimetric probe or HFM) was in both cases about $140 \mathrm{~cm}$. During the experiments, $\mathrm{U}_{\mathrm{A}}$ was varied from 200 to $1700 \mathrm{~V}$ resulting in an increase of the kinetic energy of the ions in the beam hitting the sensors. Broad beam ion sources as used in this experiments are commonly explored for surface structuring (e.g. ion beam figuring) [20] or as space thrusters.

As a second example, measurements of the energy flux have been performed in different experiments in the laboratory set-up "MicroSys". The plasma in "MicroSys" is a common capacitively coupled asymmetric rf-discharge $(13,56 \mathrm{MHz})$ which is normally used in complex (dusty) plasma experiments and for plasma processing (e.g. PECVD). The cylindrically shaped reactor has a diameter of $400 \mathrm{~mm}$, the height is $400 \mathrm{~mm}$, too. The powered rf-electrode has a diameter of $100 \mathrm{~mm}$ and can be vertically moved, the chamber walls were at ground potential. We performed some test series in the rf-discharge with an argon pressure of $10 \mathrm{~Pa}$ and an rf-power in the range from 10 to $100 \mathrm{~W}$. The thermal sensors were alternatively placed at $40 \mathrm{~mm}$ above the powered electrode. The sensor surfaces were set perpendicular to the electrodes. Although the sizes of the inserted sensors are rather small compared to the plasma volume, the perturbation of the plasma cannot always be neglected. However, this is not a serious problem, because in case of real substrates which are placed in the reactor, there is also an interaction with the surrounding plasma. Moreover, in the present study the characterization and comparison of both thermal sensors in plasma environment are of main interest - and the sizes of the sensors as well as their interaction with the plasma are quite similar.

\section{Results and discussion}

The results obtained from measurements of the energy influx in the ion beam are presented in figure 6 . At a distance of $140 \mathrm{~cm}$ from the grids of the ion source, energetic neutral argon atoms mainly contribute to the total energy influx due to charge exchange collisions. For both sensors, the measured energy influx increases with the ion energy (anode voltage) in the same manner. For energy fluxes lower than $100 \mathrm{~mW} / \mathrm{cm}^{2}$ the results are nearly the same. However, it can easily be seen in Fig. 6, that the difference between the values provided by both sensors increases with the anode voltage, e.g. with the kinetic energy of the incoming fast ions or neutrals, respectively. This difference can be explained by the design of the calorimetric probe and the calculation method of the energy flux by using the effective heat capacity of the probe. According to Stahl et al. [18] the heat capacity of the probe holder (made of Macor $®$ ) depends on the temperature of the holder itself. At high temperatures which are achieved in the energetic ion beam, this effect can induce an error of about $10 \%$. The errors for the energy influxes obtained by the HFM method are estimated from statistics, e.g. from comparison of results obtained under identical experimental conditions at different dates. The corresponding uncertainties are plotted in the graphs of Fig.6.

In figure 7 the temporal evolution of the HFM voltage (Fig. 7a) and the temporal evolution of the temperature $\mathrm{T}_{\mathrm{S}}(\mathrm{t})$ for the calorimetric probe (Fig.7b) in the asymmetric RF discharge are presented. Several measurement cycles have been performed by changing the discharge power stepwise from 10 to $100 \mathrm{~W}$. In both measurement methods the heating and 
the cooling regimes can be seen according to the RF power. Similar as for the measurements performed in the ion beam also for the experiments in the RF plasma the shape of the HFM signal is well defined (Fig.7a). As a response of the heating by the plasma at applied RF power the HFM voltage increases fastly and reaches a steady state during the plasma process. Finally, when the plasma is switched off, the voltage sharply decreases. The offset value (close to $2.5 \mu \mathrm{V}$ ) which can be recognized between two applied powers is again due to radiative transfer between the chamber and the HFM at $5^{\circ} \mathrm{C}$, as explained below. To determine the energy influx due to the RF plasma only the voltage difference between the offset and the plasma-on signal has been taken into consideration.

Due to the sensitivity of the thermopile (thin film design) the noise on the HFM voltage signal is very low, even at low energy flux density values. Consequently, the corresponding energy influxes are determined with minor errors. In comparison, the signals obtained by the calorimetric probe for RF power less than $60 \mathrm{~W}$ (e.g. energy influxes less than $35 \mathrm{~mW} / \mathrm{cm}^{2}$ ) are rather noisy. This fact induces an additional source of error.

In figure 8 the energy influxes determined by the calorimetric probe and the HFM in the RF plasma are presented. As expected, for both sensors the energy influx increases almost linearly with the RF power. Nevertheless, the values obtained by the HFM are about $40 \%$ higher than those obtained by the calorimetric probe.

Obviously, in contrast to the almost identical results obtained by both sensors during the interaction with a highly energetic and anisotropic ion beam, in case of a rather small and isotropic energy influx by the RF plasma, there is a discrepancy between the values provided by the sensors. Three possible explanations for this discrepancy may be considered: (i) the difference between the calorimetric probe temperature at the beginning and at the end of the experiment was about $6^{\circ} \mathrm{C}$ (see fig. 7b). Thus, the residual probe temperature slowly increased which may influence the energy flux calculated value. (ii) Another reason for this difference may be the presence of the RF filter in the acquisition system of the calorimetric probe which was necessary for RF plasma application. This problem could be solved by calibrating the probe in the presence of the filter. (iii) Finally, there could be speculated about an additional reason of the discrepancy in the measured energy influxes in the RF plasma. Although both sensors detect only average fluxes due to their sizes of a few millimeters the disturbance as well as the detection volume are somewhat different. Since the diameter of the calorimetric probe is of $20 \mathrm{~mm}$ (compared to $6 \mathrm{~mm}$ for the HFM), local inhomogeneities of the plasma characteristics may lead to the observed differences. Thus, a tiny spatial variation of the energy flux density in a plasma of such a rather weak rf-discharge may not be detected. Nevertheless, even if small differences between the measurements of the sensors appear in the case of RF plasma, the results obtained with both sensors in this low value range of energy influxes are in good agreement.

\section{Conclusion}

In this work two different sensors for the measurement of the energy influx in low pressure plasmas have been compared. The commercial thermopile sensor (HFM) allows for a direct measurement of the energy influx, whereas in the case of the calorimetric probe the energy influx has to be calculated from a temperature characteristics $T_{S}(t)$ measured by the probe. Two campaigns of experiments were performed at IEAP Kiel. Firstly, both sensors were placed in front of an energetic ion beam and, secondly, in an asymmetric RF discharge. Although their working principles and calibration methods are different, the results obtained for the related energy fluxes are in good agreement. 
The main advantage of the calorimetric probe is its low cost, simplicity and sturdiness. It has been shown that this probe provides reliable results in high energy plasma processes as plasma jet, ion beam and magnetron discharge $[2,14,20,21]$. However, the energy influx is obtained from the evaluation of the measured temperature curves. This evaluation method can cause errors of about $10 \%$. The method requires also a certain acquisition time (seconds to minutes) which can be a problem to detect low energy contributions or transient energy transfer processes. Thus, the calorimetric probe is a less expensive and powerful tool for the measurements of total energy influxes when detection of fast transfer processes is not required.

The design of the Heat Flux Microsensor is based on thin film technique. Therefore, the main drawbacks are its high cost and fragility. High energy influxes can damage the thermopile, but this problem has been solved by positioning a substrate (copper) in front of the sensor. The HFM is characterized by a very good time resolution which can even be increased by the ablation of the black coating (zynolithe) and the optimization of the acquisition system. It has been previously demonstrated that this fast response allows for the separation of energetic contributions depending on their kinetics [17]. Consequently, the HFM is an interesting tool to separate energetic contributions and detect low energy influxes. With an optimized time resolution it could also be an useful diagnostic for the characterization of pulsed plasma processes (e.g. HIPPMS).

Acknowledgements

This work was partly supported by the Deutsche Forschungsgemeinschaft under SFB TR 24 / B4 and the Agence Nationale pour la Recherche (ANR, projet ANR-06-JCJC-0147). 


\section{References}

[1] J. Ding, J.S. Jenq, G.H. Kim, H.L. Maynard, J.S. Hamers, N. Hershkowitz and J.W. Taylor 1993 J. Vac. Sci. Technol. A11 1283

[2] H. Kersten, H. Deutsch, H. Steffen, G.M.W. Kroesen and R. Hippler 2001 Vacuum 63385

[3] S.D. Ekpe and S.K. Dew 2003 J. Vac. Sci. Technol. 21476

[4] J.C. Moreno-Marin, I. Abril, A. Gras-Marti, V. Konoplev, J.J. Jimenez-Rodriguez and A.M.C. Perez-Martin 1994 Vacuum 451135

[5] J. A.Thornton 1978 Thin Solid Film 5423

[6] R. Wendt, K. Ellmer and K.Wiesemann 1997 J. Appl. Phys. 822116

[7] J.A. Thornton 1978 Thin Solid Film 5423

[8] D.J. Ball 1972 J. Appl. Phys. 43(7) 3047

[9] R. Gardon 1952 The Rev. of Sci. Instrum. 24(5) 366

[10] T.P. Drüsedau, T. Bock, T.-M. John, F. Klabunde and W. Eckstein 1999 J. Vac. Sci. Technol. A 15(5) 2896

[11] T.P. Drüsedau, M. Löhmann, F. Klabunde an T.-M. John 2000 Surf. Coat. Technol. 133-134 126

[12] H. Kersten, R. Wiese, M. Hannemann, A. Kapitov, F. Scholze, H. Neumann and R. Hippler 2005 Surf. Coat. Technol. 200809

[13] K. Ellmer and R. Mientus 1999 Surf. Coat. Technol. 116-119 1102

[14] M. Wolter, M. Stahl and H. Kersten 2009 Plasma Processes and Polymers 6(S1) S626

[15] R. Dussart, A. L. Thomann, L. E. Pichon, L. Bedra, N. Semmar, P. Lefaucheux, Dussart R, Thomann A L, Pichon L E, Bedra L, Semmar N, Lefaucheux P, Mathias J and Tessier Y 2008 Appl. Phys.Lett. 93131502

[16] A. Thomann, N. Semmar, R. Dussart, J. Mathias and V. Lang 2006 Rev. Sci. Instrum. 77033501

[17] L. Bedra, A.L. Thomann, N. Semmar, R. Dussart and J. Mathias 2010 J. Phys. D: Appl. Phys.. 43 065202

[18] M. Stahl, T. Trottenberg, and H. Kersten 2010 Rev. Sci. Instrum. 81023504

[19] Murthy A V, Tsai B K and Saunders R D 2000 J. Res. Natl. Inst. Stand. Technol. 105293

[20] V. Schneider, T. Trottenberg, I. Teliban and H. Kersten Rev. Sci. Instrum. 81013503

[21] D. Lundin, M. Stahl, H. Kersten and U. Helmersson 2009 J. Phys. D. Appl. Phys. 42185202 


\section{Figure captions}

Figure 1: Scheme of the HFM (a) and photograph of the active area of the HFM taken with a binocular (b).

Figure 2: Shape of the signal (thermo-voltage) obtained if the HFM is exposed to an energy flux in an asymmetric RF discharge for a pressure of $10 \mathrm{~Pa}$ and an RF power of $70 \mathrm{~W}$.

Figure 3: Calibration curve of the HFM with and without $\mathrm{Cu}$ sample.

Figure 4: Scheme of the calorimetric probe

Figure 5: Temperature characteristics $\mathrm{T}_{\mathrm{S}}(\mathrm{t})$ measured by the calorimetric probe in asymmetric $\mathrm{RF}$ discharge at a pressure of $10 \mathrm{~Pa}$ and an $\mathrm{RF}$ power of $70 \mathrm{~W}$.

Figure 6: Measured energy influx in dependence on the anode voltage (kinetic ion energy).The HFM or the calorimetric probe, respectively, were placed in the centre of the ion beam at a distance of $140 \mathrm{~cm}$ from the source.

Figure 7: a) Temporal evolution of the HFM voltage in a asymmetric RF discharge for different input powers; b) temporal evolution of the temperature for the calorimetric probe in an asymmetric RF discharge for different input powers.

Figure 8: Energy influx plotted against the RF power when the HFM or the calorimetric probe, respectively, were placed in the asymmetric RF discharge. 
Figure 1

Thermocouple junctions

a)

Zynolite coating thermopile

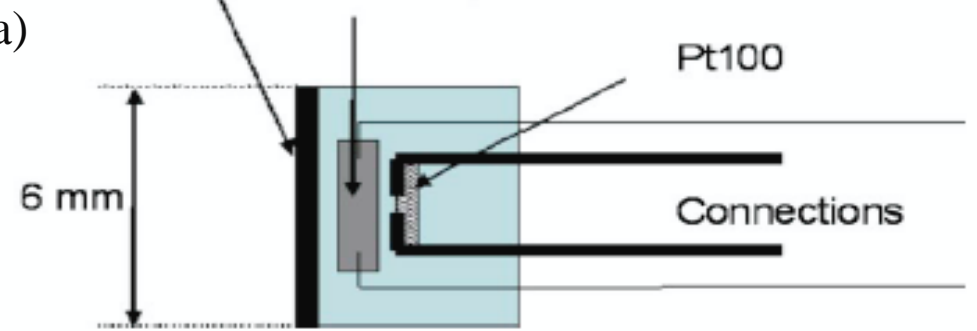

b)

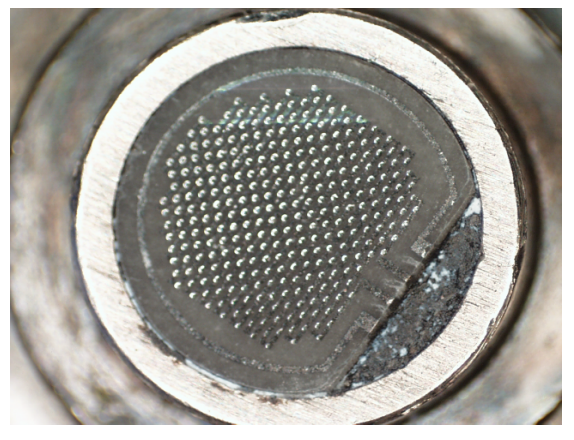


Figure 2

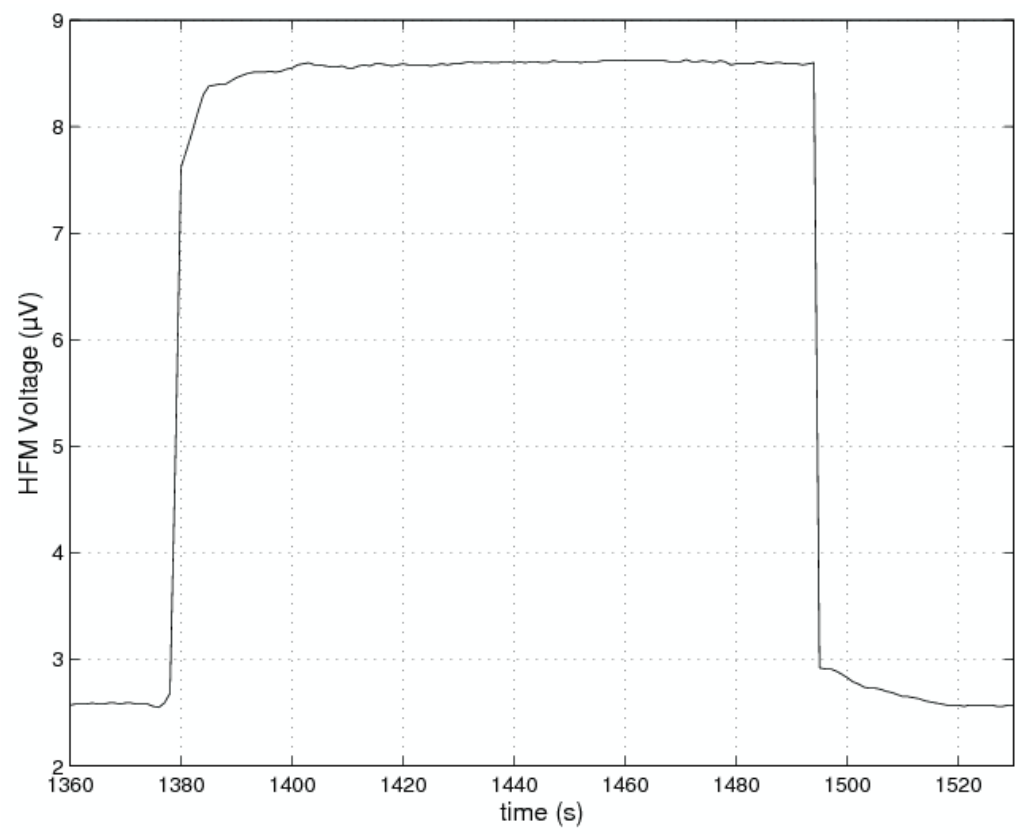


Figure 3

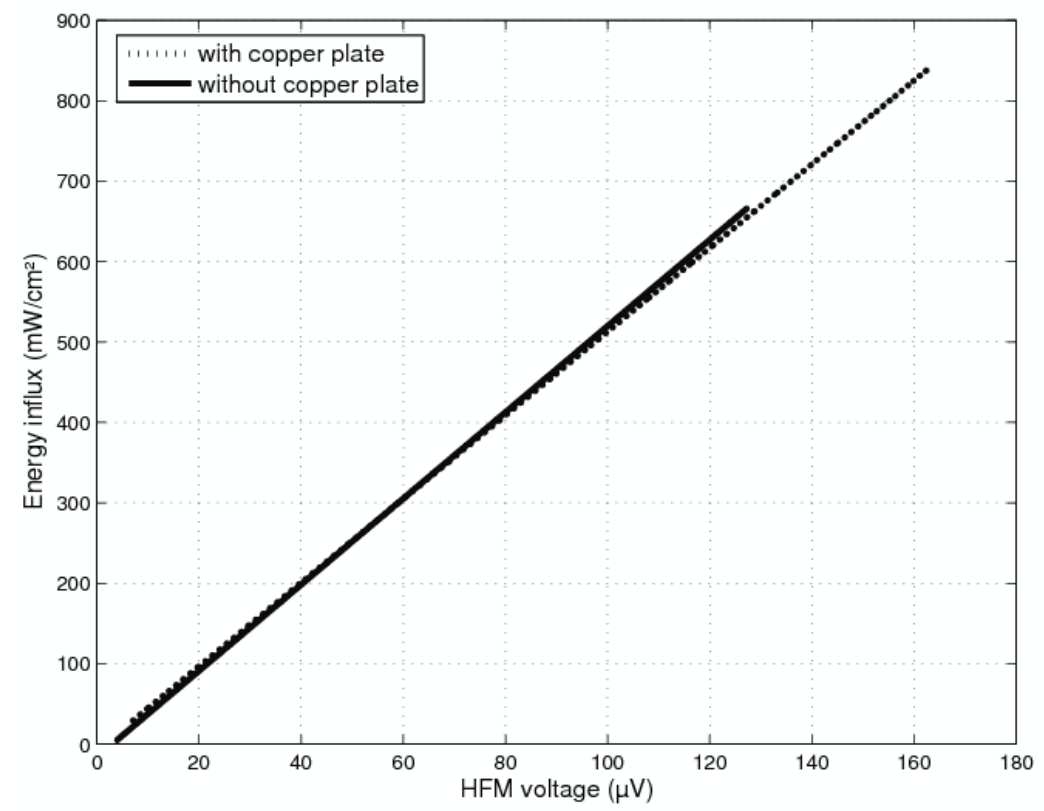


Figure 4

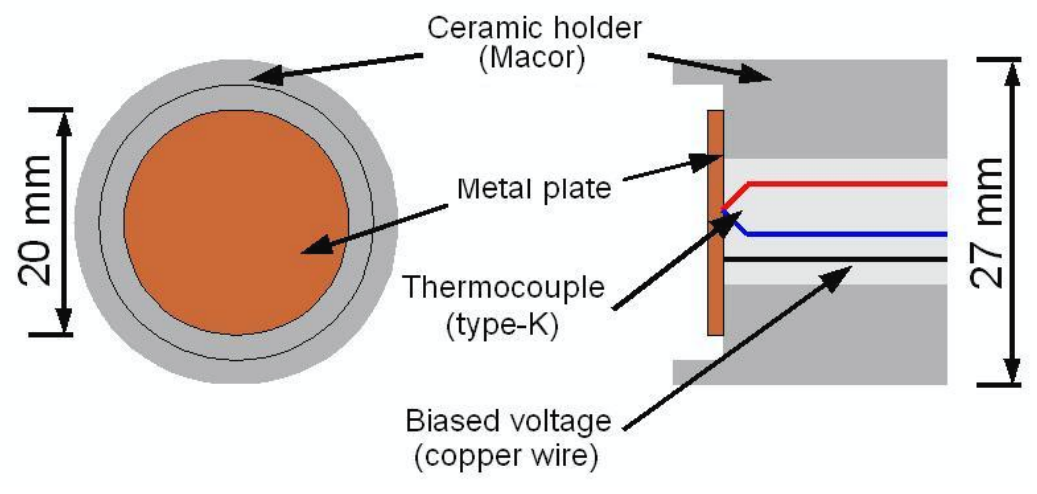


Figure 5

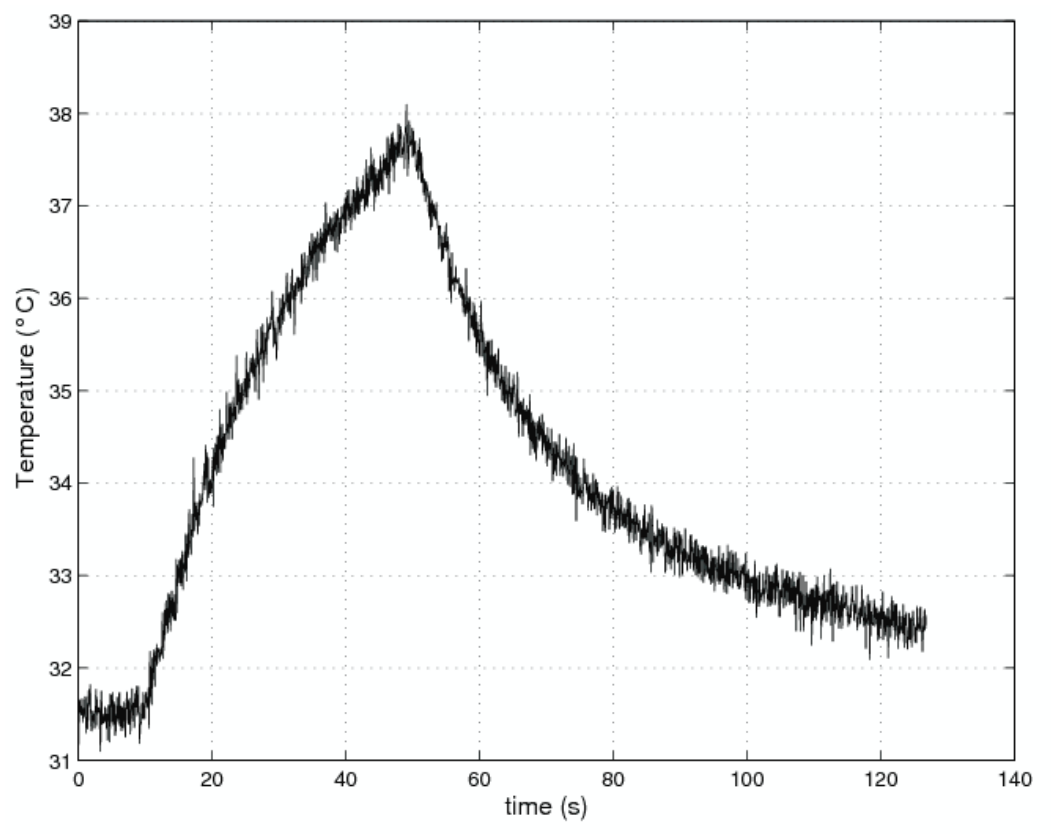


Figure 6

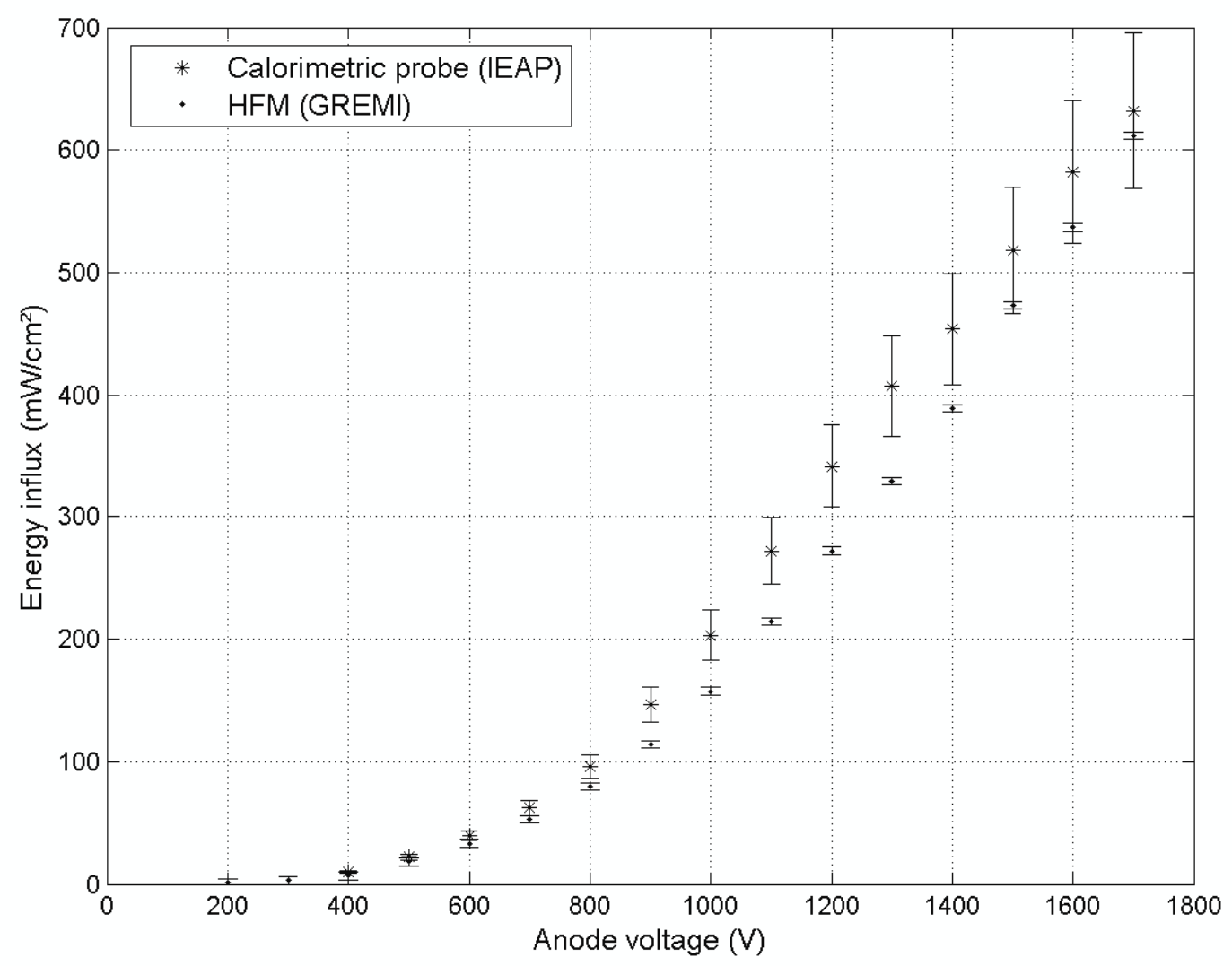


Figure 7
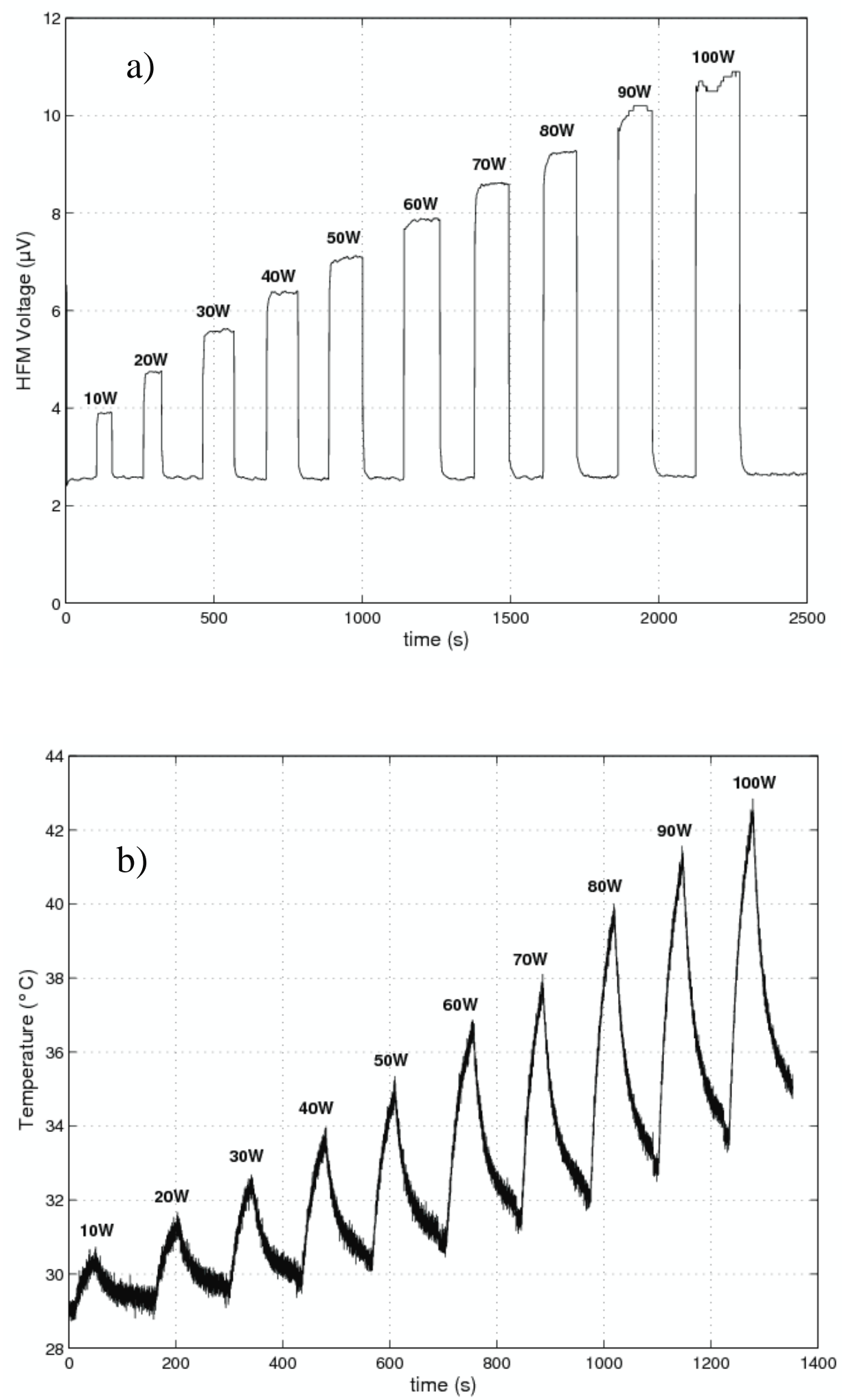
Figure 8

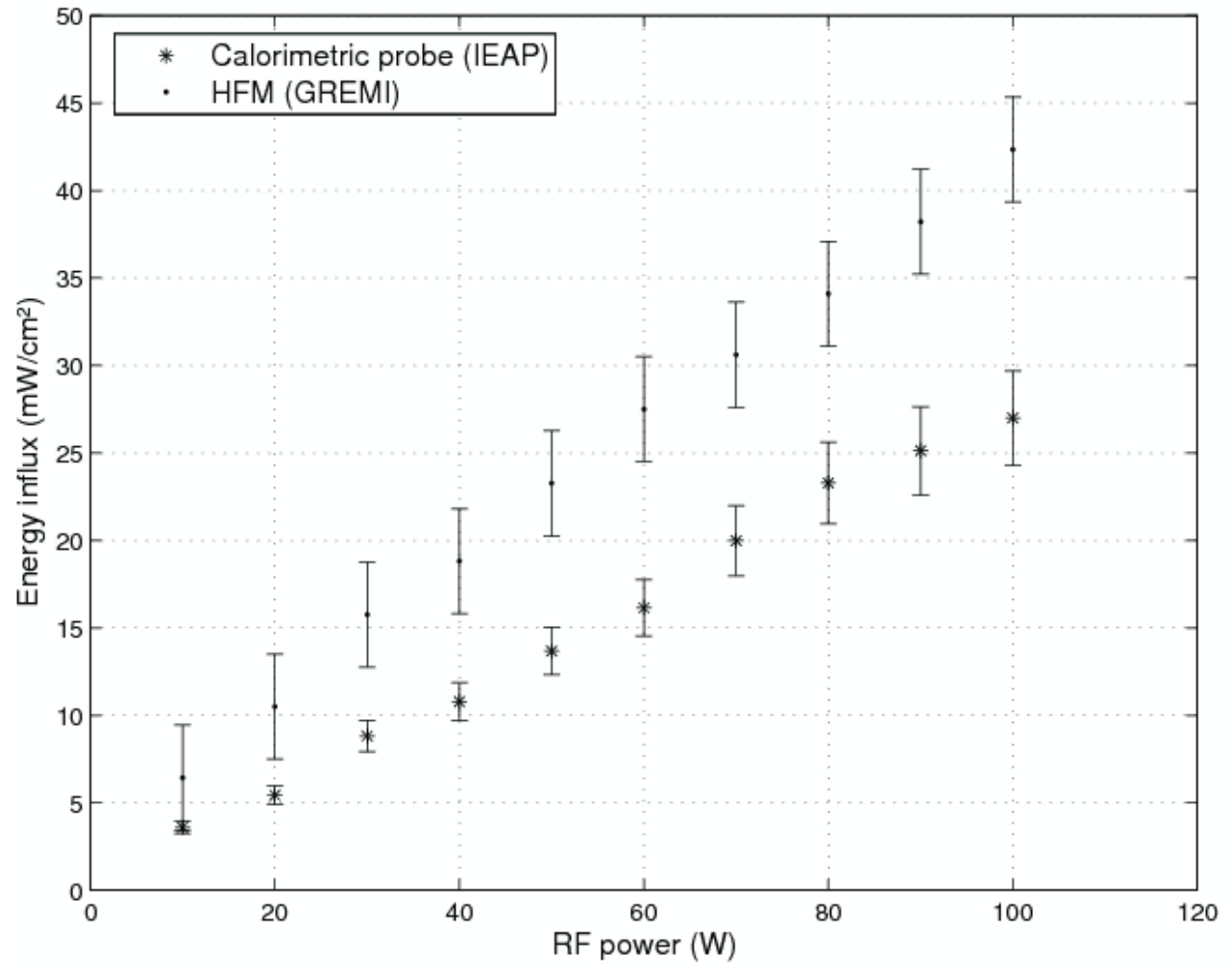

